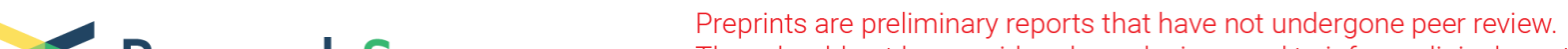 $\begin{array}{ll}\text { Research Square } & \text { They should not be considered conclusive, used to inform clinical practice, } \\ \text { or referenced by the media as validated information. }\end{array}$
}

\section{Somatic Genomic Defects And Tumor Progression In NF1-associated Tumors}

\section{Yah-Huei Wu-Chou ( $\nabla$ yhwc8876@gmail.com )}

Department of Medical Research, Chang Gung Memorial Hospital at Linko, Taoyuan, Taiwan https://orcid.org/0000-0003-2522-8215

\section{Tzu-Chao Hung}

Chang Gung Memorial Hospital Linkou Main Branch: Chang Gung Memorial Hospital

\section{Yin-Ting Lin}

Chang Gung Memorial Hospital Linkou Main Branch: Chang Gung Memorial Hospital

\section{Chih-Hung Lin}

Chang Gung Memorial Hospital Linkou Main Branch: Chang Gung Memorial Hospital

\section{Chung-Chih Yu}

Chang Gung Memorial Hospital Linkou Main Branch: Chang Gung Memorial Hospital

\section{Shih-Chiang Huang}

Chang Gung Memorial Hospital Linkou Main Branch: Chang Gung Memorial Hospital

\section{Kuo-Ting Chen}

Chang Gung Memorial Hospital Linkou Main Branch: Chang Gung Memorial Hospital

\section{Yu-Ray Chen}

Chang Gung Memorial Hospital Linkou Main Branch: Chang Gung Memorial Hospital

\section{Research article}

Keywords: NF1-associated tumors, targeted NGS panel analysis, copy number variations, tumor pathology, neurofibromatosis

Posted Date: January 15th, 2021

DOI: https://doi.org/10.21203/rs.3.rs-144193/v1

License: (c) (1) This work is licensed under a Creative Commons Attribution 4.0 International License. Read Full License 


\section{Abstract}

Background: Neurofibromatosis type 1 (NF1) is a common inherited tumor predisposition syndrome. Many genetic and biochemical evidences considered NF1 gene as a tumor suppressor gene that inactivation of both NF1 alleles would lead to tumorigenesis. However, the clinical evaluation and early detection of malignant transformation from plexiform neurofibromas is often challenging.

Methods: We used Affymetrix's OncoScan FFPE Assay to record copy number gains and losses of common cancer genes in 24 NF1-associated tumors and compared with their NF1 mutation status. In addition, we carried out immunohistochemical studies of NF1, SUZ12 and H3K27me3 to evaluate gene expression in NF1-associated tumors to elucidate their potential roles during neurofibromatosis.

Results: The most common Copy number alterations (CNAs) identified in this study were copy number loss (bi-allelic loss) of NF1 $(\mathrm{N}=8)$ and SUZ12 $(\mathrm{N}=7)$. Many concurrent alterations of both NF1 and SUZ12 were also observed in these NF1-associated tumors. We found that the molecular events contributing to these tumors (both PNFs and MPNST) are very heterogeneous and not all MPNST harbour biallelic NF1 inactivation. We also observed some unexplained features need to be clarified in a set of NF1-associated tumors with the genetic results and NF1, SUZ12 and H3K27me3 proteins expression.

Conclusions: We suggest that dividing NF1-associated tumors into subsets by the genetic spectrum of coordinate signaling pathway and tumor pathology may have practical implications for further choice of therapies.

\section{Background}

Neurofibromatosis type 1 (NF1) is dominantly inherited tumor predisposition syndrome that are highly variable and affect several organ system. The majority of patients with NF1 develop cutaneous neurofibromas (cNFs), and approximately one third develop benign plexiform neurofibromas (pNFs) during their lifetime. The occurrence of multiple benign dermal neurofibromas and/or plexiform neurofibromas arising from peripheral nerve sheaths, is the clinical key feature of Neurofibromatosis type $1[1,2]$. Approximately $8-13 \%$ of NF1 patients has a lifetime risk for developing malignant peripheral nerve sheath tumors (MPNSTs) which generally arise by malignant progression of preexisting plexiform neurofibromas $[3,4]$.

The cause of NF1-associated tumor formation is largely explained by the underlying genetic defect of the NF1 gene which encodes neurofibromin, a RAS-GTPase-activating protein (RAS-GAP) that is directly involved in the regulation of Ras signaling [5-8]. Many genetic and biochemical evidences considered NF1 a tumor suppressor gene such that biallelic NF1 inactivation is the critical events in the process of NF1-associated tumorigenesis [7, 8]. In accordance with Knudson's two-hit hypothesis, somatic NF1 inactivation has been demonstrated in both benign neurofibromas and malignant peripheral nerve sheath tumors [9], frequently evidenced by loss of heterozygosity (LOH) [10-12]. Loss of neurofibromin function may be necessary for neurofibroma formation, however, it is not sufficient for transformation into a 
malignant MPNST [13]. A number of studies have attempted to identify tumor-specific molecular signatures beyond NF1 for MPNSTs [14-19] and benign neurofibromas [17, 20]. MPNST cells harbor highly rearranged genomic architecture and have frequent loss of either CDKN2A/B or TP53 in the MPNST tumor cells, either via somatic copy number alterations (SCNAs) or single nucleotide variants (SNVs). Integrated genomic and molecular studies have later provide evidence that many chromatin modification genes may also involve in NF1 tumor progression. Loss of function mutations in the Polycomb repressive complex 2 (PRC2) component genes, such as SUZ12 or EED, are now known to be very common in MPNSTs, occurring in $23 \sim 70 \%$ of NF1-associated cases and more than $90 \%$ of sporadic cases [19, 21-23]. These PRC2 inactivation finally leads to loss of trimethylation at lysine 27 of histone H3 (H3K27me3) for regulate RAS-driven transcription [24, 25].

In this study, we present the genetic spectrum of a cohort NF1-associated tumors through a NGS targeted gene sequencing approach and a copy number analysis of NF1 and SUZ12 in NF1-associated tumors. Meanwhile, we have accessed tumor immunohistochemistry of NF1, SUZ12 and H3K27me3 to evaluate their essential roles during neurofibromatosis.

\section{Methods}

\section{Study subjects and sample preparations}

NF1 patients who satisfied the National Institutes of Health diagnostic criteria (NIH Consensus Statement) and who had developed either benign or malignant tumors were recruited. Tumor specimens were obtained from patients who undergo surgical resection at the Chang Gung Memorial Hospital. All patients provided informed written consent for their tissues to be used in this study, which was approved by the Chang Gung Medical Foundation Institutional Review Board. The corresponding clinical data were collected and used in accordance with proved protocols from Chang Gung Medical Foundation Institutional Review Board. Patient-matched lymphocyte DNA were also included to determine the germline event(s) of these NF1-associated tumors. Genomic DNA from archived tumor samples were prepared by PUREGENE DNA purification kit fit from GENTRA according to the manufacturer's instruction.

\section{Targeted gene sequencing using the IT-PGM Sequencing system}

A custom designed NGS panel including five common NF1-related genes: NF1 (NM_000267, 17q11.2), SPRED1 (NM_152594, 15q14), KRAS (NM_004985, 12p12.1), BRAF (NM_004333, 7q34), and TP53

(NM_000546, 17p13.1) was performed for the mutation analysis in this study. The total length is $32.3 \mathrm{~kb}$, 296 amplicons and the coverage is 507x. Template preparation and emulsion PCR, and lon Sphere Particles (ISP) enrichment were performed on an lon One Touch System by using the lon OneTouch ${ }^{\text {TM }} 200$ Template Kit Version 2.0 (Life Technologies) according to the manufacturer's instructions. The raw PGM data were processed with Torrent Suite v3.4.2 (Life Technologies) to generate sequence reads filtered by the pipeline software quality controls. The Integrative Genomics Viewer16 (Broad Institute, Cambridge, Massachusetts) was then used to visualize variants against the reference genome and for the analysis of 
depth coverage, sequence quality, and variant identification. Variant frequencies $>1 \%$ in the dbSNP-Asian database were further filtered.

\section{PCR amplification and Sanger sequencing verification}

Fragments with putative NF1 mutations or potential mutations were amplified by PCR and sequenced with Applied Biosystems 3730 Genetic Analyzer in DNA Sequencing Service of Chang Gung Memorial Hospital. A Standard Sanger sequencing was also performed exon by exon for SUZ12. PCR amplification were carried out under standard conditions with $30 \mathrm{PCR}$ cycles and $55^{\circ}-60^{\circ} \mathrm{C}$ annealing. PCR products will then be sequenced using the Big Dye Terminator cycle sequencing kit (Life Technologies) according to manufacturer's cycling conditions. Sequence alignments and analysis were further performed using the Autoassembler computer program (Life Technologies).

\section{Molecular inversion probe (MIP) DNA array analysis}

The Affymetrix OncoScan Assay utilizing the Molecular Inversion Probe (MIP) technology was performed for larger copy number alterations and loss of heterozygosity (LOH). This OncoScan assay contains more than 300,000 copy number and single nucleotide polymorphism (SNP) oligonucleotide probes with a median probe spacing of 4,200 kilobases $(\mathrm{kb})$, with much denser coverage within known cancer genes. Twenty-four available tumor DNA samples (80 $\mathrm{ng}$ genomic DNA) was performed following protocols provided by manufacturer (Affymetrix, Santa Clara, CA, USA). Briefly, samples were split to separate (A/T) and (G/C) channels. After circularization, MIPs were linearized, cleaved and then amplified by PCR. Amplicons were then cleaved into two fragments (44 bp) with Haell. DNA fragments were subsequently hybridized to OncoScan ${ }^{\circledR}$ arrays at $49^{\circ} \mathrm{C}$ and $60 \mathrm{rpm}$ for $16-18 \mathrm{~h}$. The hybridized array were then washed, stained and scanned through GeneChip ${ }^{\circledR}$ scanner $30007 \mathrm{G}$ (Affymetrix, CA). Array fluorescence intensity data (CEL files), generated by Affymetrix ${ }^{\circledR}$ GeneChip ${ }^{\circledR}$ Command Console ${ }^{\circledR}$ (AGCC, Affymetrix) Software version 1.1 were processed to produce OSCHP files and QC metrics. Samples passing QC criteria (MAPD $\leq 0.3$, ndSNPQC $\geq 26$ ) were further analyzed through tumor Scan (TuScan) and BioDiscovery's SNPFASST2 algorithm using the Nexus Express for Oncoscan software 3.0 (Biodiscovery, Hawthorne, CA) and whole chromosome gains and losses and copy number aberrations (deletions and duplications) were determined.

\section{Protein expression through immunohistochemistry in different NF1- associated tumors}

Paraffin-embedded surgical resection specimens were retrospectively collected from the archives of the Tissue Bank at Department of Pathology, Chang Gung Memorial Hospital, for subsequent molecular analysis. Five micrometer sections mounted onto poly-L-lysine coated slides were deparaffinized and rehydrated in graded alcohols and distilled water. Endogenous peroxidase activity were quenched by incubation in $3 \%$ hydrogen peroxide in methanol for $20 \mathrm{~min}$. Antigen retrieval were done by microwaving at high power for two cycles of 5 min each with a 10 min break between cycles in citrate buffer at pH 6.0. Slides were incubated with each primary antibodies at $4^{\circ} \mathrm{C}$ overnight. A mouse monoclonal antibody NF1 (A-12) (Sc-74445; Santa Cruz Biotechnology, Santa Cruz, CA), targeting amino acids 1-300 of the NF1 
protein, was used as NF1 primary antibody. The SUZ12-antibodies used in this study was mouse monoclonal antibody against clone SUZ220A (ab126577; Abcam, Cambridge, UK).

Immunohistochemistry for H3K27me3 was performed using a rabbit polyclonal antibody directed against the trimethylated lysine 27 residue of the N-terminal portion of histone H3 (1:500 dilution; Cat.\# 07-449; Millipore, Billerica, MA, USA). Following the incubation of primary antibodies, these slides were then incubated with horseradish peroxidase-avidin-biotin complex (Vectastain ABC Elite, Vector Laboratories). The enzyme-binding site were visualized by 3, 3-diaminobenzidine (DAB-kit, Vector Laboratories). Nonspecific binding of secondary antibody were then blocked by incubating in 10\% normal horse serum in PBS containing $1 \%$ BSA. Finally, the slides were counterstained with hematoxylin and dehydrated in graded alcohol; air dried, and mounted using Resin-based mounting medium under coverslips. The results of immunoreactivity of each antibody were evaluated by pathologist and scored according to the percentage of tumor cells with positive nuclear immunostaining and staining intensity. Each sample was scored as follows: $\otimes$, negative; $1 \rrbracket, 5 \%$ positive cells; $2 \bigotimes, 6-50 \%$ positive cells; $3 \bigotimes,>50 \%$ positive cells.

\section{Statistical analysis}

SPSS 13.0 software package was used for all statistical analyses. For evaluation of the difference in percentage of numbers of mutation, the Mann-Whitney $U$ test was performed. A $P$ value of $<0.05$ was considered to be statistical significance.

\section{Results}

\section{NF1 mutation and copy number alterations in 24 NF1-associated tumors}

A targeted gene sequencing panel with five NF1-related genes include NF1, SPRED1, KRAS, BRAF and $p 53$ was performed in twenty-four NF1-associated tumors from unrelated patients. In total, we found genetic alterations of NF1 gene in 23/24 neurofibromas including 3 missense, 13 nonsense, Single splice site mutations, and 6 frameshift mutations. Four tumors which did not show NF1 mutations in NGS screening were subjected to confirm for NF1 single- and multi-exon deletions-duplications by P081-P082 MLPA analyses (Table 1). In the copy number variation analysis, we found that the most common copy number alterations (CNAs) identified in this study were copy number loss (bi-allelic loss) of NF1 (8 of 24 tumors) and SUZ12 (6 of 24 tumors). We have also detected the occurrence of parallel DNA alterations in these two genes (Wu-2, Wu-4, Wu-22, Wu-23, and Wu-45). The co-localization of these two genes on $17 q 11$ might explain this coincidence of the alterations (Fig.1).

\section{Pathology and immunohistochemistry for 24 NF1-associated tumors}

A cohort of 24 NF1-associated tumors (19 neurofibromas, 2 atypical neurofibroma and 3 malignant peripheral nerve sheath tumors) from unrelated patients having a clinical diagnosis of neurofibromatosis 1 was analyzed in this study. PNFs and MPNST normally show drastic differences in histological features. Sometimes it still difficult to distinguish between the borderline tumors based on the traditional clinic pathology. Diffuse and plexiform neurofibromas (pNFs) are usually associated with major nerve 
trunks and tortuous plexi of Schwann cells and axons embedded in stroma and show interlaced bundles of elongated cells with wavy nuclei associated with wire-like strands of collagen. MPNSTs are characterized by intersecting fascicles of monotonous spindle cells with hyperchromatic nuclei and high mitotic counts with focal areas of necrosis. Atypical neurofibromas (ANF) is later hypothesized to be a premalignant lesion and pathologically defined recently by their increased variable cellularity, cytological atypia, and more pronounced fascicular growth patterns seen in MPNST (Table 2). We then employed immunohistochemistry to evaluated NF1, SUZ12 and H3K27me3 expression levels on formalinfixed/paraffin-embedded samples of all 24 available NF1-associated Tumors. Briefly, NF1 was strongly expressed in approximately $84.2 \%(16 / 19)$ of neurofibromas with a somatic or constitutional NF1 mutation, while only weak expression in the atypical neurofibroma and malignant peripheral nerve sheath tumor. For the SUZ12, most NF1-associated tumors showed weak to moderate staining intensity (Fig 2). For the H3K27me3, most available Tumors showed heterogeneous retention of H3K27 trimethylation. Only one malignant peripheral nerve sheath tumors demonstrated complete loss of staining with the anti-H3K27me3 polyclonal antibody (Fig 3). All these results of the immunohistochemical analysis are summarized in Tables 3.

\section{Discussion}

The hallmark feature of NF1 is the occurrence of multiple benign peripheral nerve sheath tumors (neurofibromas). However, the clinical evaluation and characterization of NF1-associated tumors is often challenging. In this study, we report the utilization of Affymetrix's OncoScan FFPE Assay to record copy number gains and losses in 24 NF1-associated tumors and compared with their NF1 mutation status. The most common Copy number alterations identified in this study were copy number loss of NF1 and SUZ12. We have also observed the occurrence of parallel DNA alterations in these two genes. In addition, we carried out immunohistochemical studies of NF1, SUZ12 and H3K27me3 to evaluate gene expression to elucidate their potential roles during neurofibromatosis. We found that the molecular events contributing to these tumors (both PNFs and MPNST) are very heterogeneous and not all MPNST harbour biallelic NF1 inactivation.

MPNSTs typically arise from a preexisting benign plexiform neurofibroma and have a poor prognosis. Accumulated evidence has suggests that NF1 loss is necessary but not sufficient for MPNST development. In addition to NF1 loss, MPNSTs have frequent loss of either CDKN2A or TP53 [19, 20]. CDKN2A deletions were observed in the majority of aNFs, a clear transition from a benign-atypical neurofibroma toward an intermediate-grade MPNST, by both histopathology and genetic analysis [26-30]. These mutations are rarely found in benign neurofibromas [19, 22]. A cooperative role of SUZ12 or EED inactivation, along with NF1, TP53, and CDKN2A loss-of-function, has been proposed as a key event in the development of malignancy. Recent studies have further suggested that loss of H3K27me3, which H3K27me3 represents an important epigenetic mediators of PRC2 function of chromatin regulation, may serve as a useful diagnostic marker in the distinction of malignant peripheral nerve sheath tumor from PNF [31-35]. However, we found that the molecular events contributing to these tumors (both PNFs and MPNST) are very heterogeneous and not all MPNST harbour biallelic NF1 inactivation. We also observed 
some unexplained features need to be clarified in a set of NF1-associated tumors with the genetic results and their NF1, SUZ12 and H3K27me3 proteins expression. As it has been proposed that diverse drivers of Ras-activating mutations might exist in MPNST and seem to be context dependent $[19,24,25]$. Although our sample size may not have sufficient power to detect true associations which exist among the candidate target genes with different types of neurofibromas, we believed that the validation of novel markers, identified through translational research efforts, and their incorporation into existing pathological-diagnostic system, will lead us to better refine current classification for distinct prognostic or therapeutic implications.

\section{Conclusion}

The clinical evaluation and early characterization of NF1-associated tumors is often challenging. Although PNFs and MPNST normally show drastic differences in histopathological features, sometimes it still difficult to distinguish between the borderline tumors based on the traditional pathology. We suggest that dividing NF1-associated tumors into subsets by the genetic spectrum of coordinate signaling pathway and tumor pathology may have practical implications for further choice of therapies.

\section{Abbreviations}

NF1: Neurofibromatosis type 1; FFPE: Formalin-fixed paraffin embedded; CANs: Copy number alterations; cNFs: cutaneous neurofibromas; pNFs: plexiform neurofibromas; MPNSTs: Malignant peripheral nerve sheath tumors; RAS-GAP: RAS-GTPase-activating protein; LOH: Loss of heterozygosity; SCNAs: Somatic copy number alterations; SNVs: single nucleotide variants; PRC2: Polycomb repressive complex 2; NGS: Next generation sequencing; MIP: Molecular inversion probe; DAB: 3, 3-diaminobenzidine.

\section{Declarations}

\section{Acknowledgements}

We are grateful to all the participating patients who made these studies possible. The authors also thank Genomic Medicine Core Laboratory and Tissue Bank at Chang Gung Memorial Hospital, Linko.

\section{Authors' contributions}

Experimental design: YW-C. Collection and assembly of data: $T H, Y L, C L, C Y, Y W-C$. Analysis and interpretation of the data: TH, CY, YW-C. Manuscript writing: CY, YW-C. Final approval of manuscript: all authors.

\section{Funding}

Dr. Wu-Chou was funded by grants from Chang Gung Research Foundation (CMRPG3E0941, CMRPG3H0581). 
Availabilityof data and materials

Data in this study are available upon request to the corresponding authors.

\section{Ethics approval and consent to participate}

This study was approved by the Chang Gung Medical Foundation Institutional Review Board, following the Helsinki Declaration and its later amendments. All patients provided informed written consent for their tumor specimens to be used in this study.

\section{Consent for publication}

Not applicable.

\section{Competing interests}

The authors declared no conflict of interest.

\section{Author details}

${ }^{1}$ Department of Medical Research, Chang Gung Memorial Hospital at Linko, Taoyuan, Taiwan.

${ }^{2}$ Department of Plastic \& Reconstructive Surgery, Chang Gung Memorial Hospital at Linko, Taoyuan, Taiwan. ${ }^{3}$ Department of Anatomic Pathology, Chang Gung Memorial Hospital at Linko, Taoyuan, Taiwan.

\section{References}

1. Boyd KP, Korf BR, Theos A. Neurofibromatosis type 1. J Am Acad Dermatol. 2009; 61: 1-14.

2. Ferner RE. The neurofibromatoses. Pract Neurol. 2010; 10(2):82-93.

3. Farid M, Demicco EG, Garcia R, Ahn L, Merola PR, Cioffi A, Maki RG. Malignant peripheral nerve sheath tumors. Oncologist. 2014; 19(2):193-201

4. Gutmann DH, Ferner RE, Listernick RH, Korf BR, Wolters PL, Johnson KJ.Neurofibromatosis type 1. Nat Rev Dis Primers. 2017; 3:17004.

5. Cichowski K, Jacks T. NF1 tumor suppressor gene function: narrowing the GAP. Cell. 2001; 104: 593604.

6. Zhu Y, Prada LF. Neurofibromin, a tumor suppressor in the nervous system. Exp Cell Res. 2001; 264: 19-28.

7. Yap YS, McPherson JR, Ong CK, Rozen SG, Teh BT, Lee AS, Callen DF. The NF1 gene revisited - from bench to bedside. Oncotarget. 2014; 5(15):5873-92.

8. Ratner N, Miller SJ. A RASopathy gene commonly mutated in cancer: the neurofibromatosis type 1 tumour suppressor. Nat RevCancer. 2015; 15(5):290-301.

9. Laycock-van Spyk S, Thomas N, Cooper DN, Upadhyaya M. Neurofibromatosis type 1-associated tumours: their somatic mutational spectrum and pathogenesis. Hum Genomics. 2011; 5(6):623-90. 
10. Steinmann K, Kluwe L, Friedrich RE, Mautner VF, Cooper DN, Kehrer-Sawatzki H. Mechanisms of loss of heterozygosity in neurofibromatosis type 1-associated plexiform neurofibromas. J Invest Derma. 2009; 129: 615-621.

11. Garcia-Linares C, Fernández-Rodríguez J, Terribas E, Mercadé J, Pros E, Benito L, et al. Dissecting loss of heterozygosity (LOH) in neurofibromatosis type 1-associated neurofibromas: importance of copy neutral LOH. Hum Mutat. 2011; 32: 78-90.

12. Thomas L, Mautner VF, Cooper DN, Upadhyaya M. Molecular heterogeneity in malignant peripheral nerve sheath tumors associated with neurofibromatosis type 1. Hum Genomics. 2012; 6:18.

13. Staedtke V, Bai RY, Blakeley JO. Cancer of the Peripheral Nerve in Neurofibromatosis Type 1. Neurotherapeutics. 2017; 14(2):298-306.

14. Lévy P, Vidaud D, Leroy K, Laurendeau I, Wechsler J, Bolasco G, et al. Molecular profiling of malignant peripheral nerve sheath tumors associated with neurofibromatosis type 1, based on large-scale realtime RT-PCR. Mol Cancer. 2004; 3:20.

15. Upadhyaya M, Spurlock G, Thomas L, Thomas NS, Richards M, Mautner VF, et al. Microarray-based copy number analysis of neurofibromatosis type-1 (NF1)-associated malignant peripheral nerve sheath tumors reveals a role for Rho-GTPase pathway genes in NF1 tumorigenesis. Hum Mutat. 2012; 33(4):763-76.

16. Rahrmann EP, Watson AL, Keng VW, Choi K, Moriarity BS, Beckmann DA, et al. Forward genetic screen for malignant peripheral nerve sheath tumor formation identifies new genes and pathways driving tumorigenesis. Nat Genet. 2013; 45(7):756-66.

17. Thomas LE, Winston J, Rad E, Mort M, Dodd KM, Tee AR, et al. Evaluation of copy number variation and gene expression in neurofibromatosis type-1-associated malignant peripheral nerve sheath tumours. Hum Genomics. 2015; 9:3.

18. Masliah-Planchon J, Pasmant E, Luscan A, Laurendeau I, Ortonne N, Hivelin M, et al. MicroRNAome profiling in benign and malignant neurofibromatosis type 1-associated nerve sheath tumors: evidences of PTEN pathway alterations in early NF1 tumorigenesis. BMC Genomics. 2013; 14:473.

19. Brohl AS, Kahen E, Yoder SJ, Teer JK, Reed DR. The genomic landscape of malignant peripheral nerve sheath tumors: diverse drivers of Ras pathway activation. Sci Rep. 2017; 7(1): 14992.

20. Pemov A, Li H, Patidar R, Hansen NF, Sindiri S, Hartley SW, et al. The primacy of NF1 loss as the driver of tumorigenesis in neurofibromatosis type 1-associated plexiform neurofibromas. Oncogene. 2017; 36(22):3168-3177.

21. Zhang M, Wang Y, Jones S, Sausen M, McMahon K, Sharma R, et al. Somatic mutations of SUZ12 in malignant peripheral nerve sheath tumors. Nat Genet. 2014; 46(11):1170-2.

22. Lee W, Teckie S, Wiesner T, Ran L, Prieto Granada CN, Lin M, et al. PRC2 is recurrently inactivated through EED or SUZ12 loss in malignant peripheral nerve sheath tumors. Nat Genet. 2014; 46(11):1227-32.

23. Sohier P, Luscan A, Lloyd A, Ashelford K, Laurendeau I, Briand-Suleau A, et al. Confirmation of mutation landscape of NF1-associated malignant peripheral nerve sheath tumors. Genes 
Chromosomes Cancer. 2017; 56(5):421-426.

24. Suresh K, Kliot T, Piunti A, Kliot M. Epigenetic mechanisms drive the progression of neurofibromas to malignant peripheral nerve sheath tumors. Surg Neurol Int. 2016; 7(Suppl 33):S797-S800.

25. Zhang X, Murray B, Mo G, Shern JF. The Role of Polycomb Repressive Complex in Malignant Peripheral Nerve Sheath Tumor. Genes (Basel). 2020; 11(3):287.

26. Rodriguez FJ, Folpe AL, Giannini C, Perry A. Pathology of peripheral nerve sheath tumors: diagnostic overview and update on selected diagnostic problems. Acta Neuropathol. 2012; 123(3):295-319.

27. Higham CS, Dombi E, Rogiers A, Bhaumik S, Pans S, Connor SEJ, et al. The characteristics of 76 atypical neurofibromas as precursors to neurofibromatosis 1 associated malignant peripheral nerve sheath tumors. Neuro Oncol. 2018; 20(6):818-825.

28. Miettinen MM, Antonescu CR, Fletcher CDM, Kim A, Lazar AJ, Quezado MM, et al. Histopathologic evaluation of atypical neurofibromatous tumors and their transformation into malignant peripheral nerve sheath tumor in patients with neurofibromatosis 1-a consensus overview. Hum Pathol. 2017; 67:1-10.

29. Pemov A, Hansen NF, Sindiri S, Patidar R, Higham CS, Dombi E, et al. Low mutation burden and frequent loss of CDKN2A/B and SMARCA2, but not PRC2, define pre-malignant neurofibromatosis type 1-associated atypical neurofibromas. Neuro Oncol. 2019; 21(8):981-92.

30. Rhodes SD, He Y, Smith A,Jiang L, Lu Q, Mund J, et al. Cdkn2a (Arf) loss drives NF1-associated atypical neurofibroma and malignant transformation. Hum Mol Genet. 2019; 28(16):2752-2762.

31. De Raedt T, Beert E, Pasmant E, Luscan A, Brems H, Ortonne N, et al. PRC2 loss amplifies Ras-driven transcription and confers sensitivity to BRD4-based therapies. Nature. 2014; 514(7521):247-51.

32. Schaefer IM, Fletcher CD, Hornick JL. Loss of H3K27 trimethylation distinguishes malignant peripheral nerve sheath tumors from histologic mimics. Mod Pathol. 2016; 29:4-13.

33. Prieto-Granada CN, Wiesner T, Messina JL, Jungbluth AA, Chi P, Antonescu CR. Loss of H3K27me3 Expression Is a Highly Sensitive Marker for Sporadic and Radiation-induced MPNST. Am J Surg Pathol. 2016; 40(4):479-89.

34. Cleven AH, Sannaa GA, Briaire-de Bruijn I, Ingram DR, van de Rijn M, Rubin BP, et al. Loss of H3K27 tri-methylation is a diagnostic marker for malignant peripheral nerve sheath tumors and an indicator for an inferior survival. Mod Pathol. 2016; 29(6):582-90.

35. Korfhage J, Lombard DB. Malignant Peripheral Nerve Sheath Tumors: From Epigenome to Bedside. Mol Cancer Res. 2019; 17(7):1417-1428.

\section{Tables}

Due to technical limitations, table 1-3 is only available as a download in the Supplemental Files section.

\section{Figures}




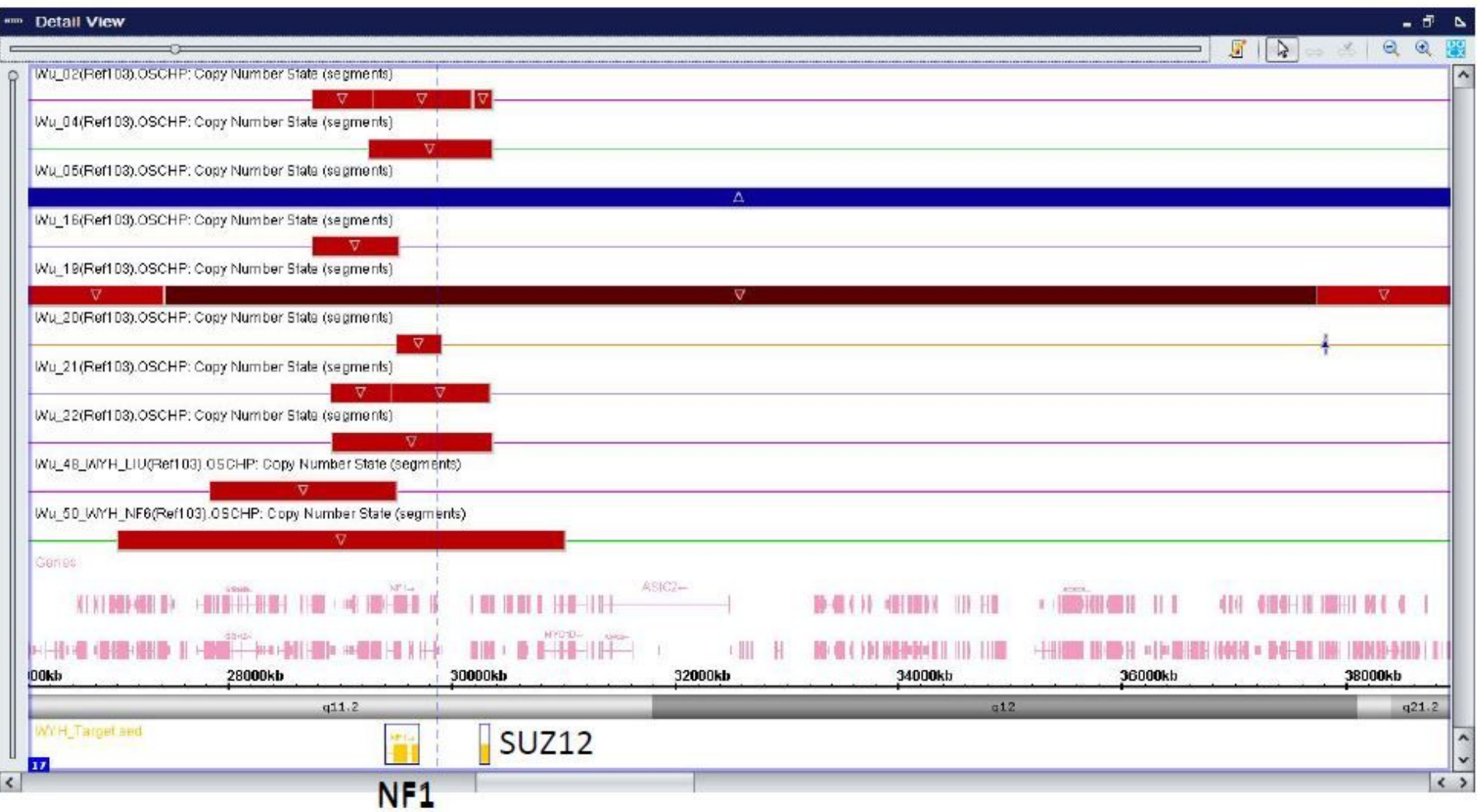

\section{Figure 1}

The Multi-sample comparison of different tumor samples with NF1 - SUZ12 concomitant deletions. Red, $\mathrm{CN}$ loss $(\mathrm{CN}<1)$; Blue, $\mathrm{CN}$ Gain $(\mathrm{CN}>2)$ 

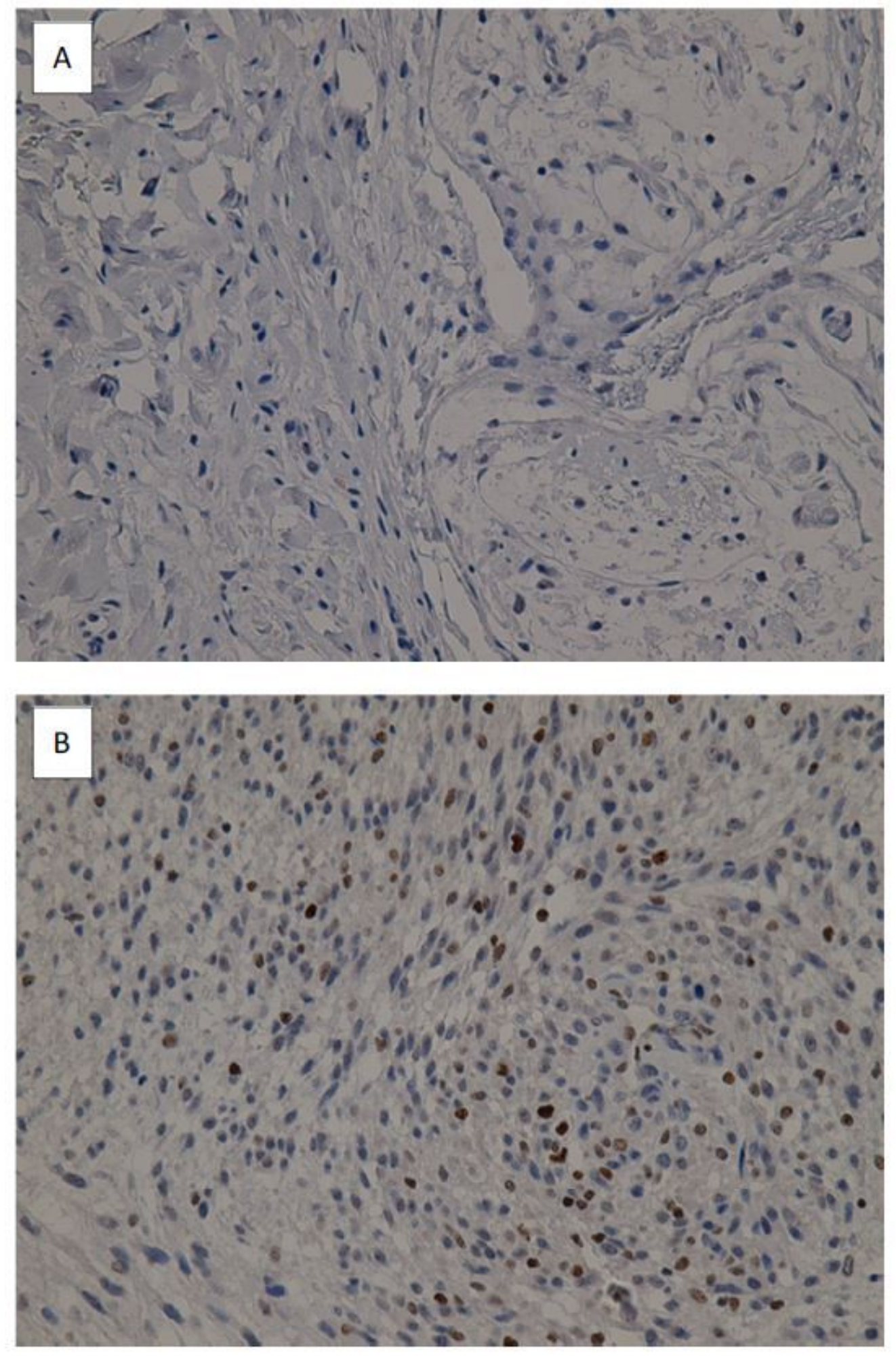

\section{Figure 2}

Immunohistochemical expression of SUZ12 in NF1-associated tumors. A. SUZ12 is lost in tumor cells in plexiform neurofibroma (SUZ12 loss) B. SUZ12 stain is retained in plexiform neurofibroma (no SUZ12 loss) 

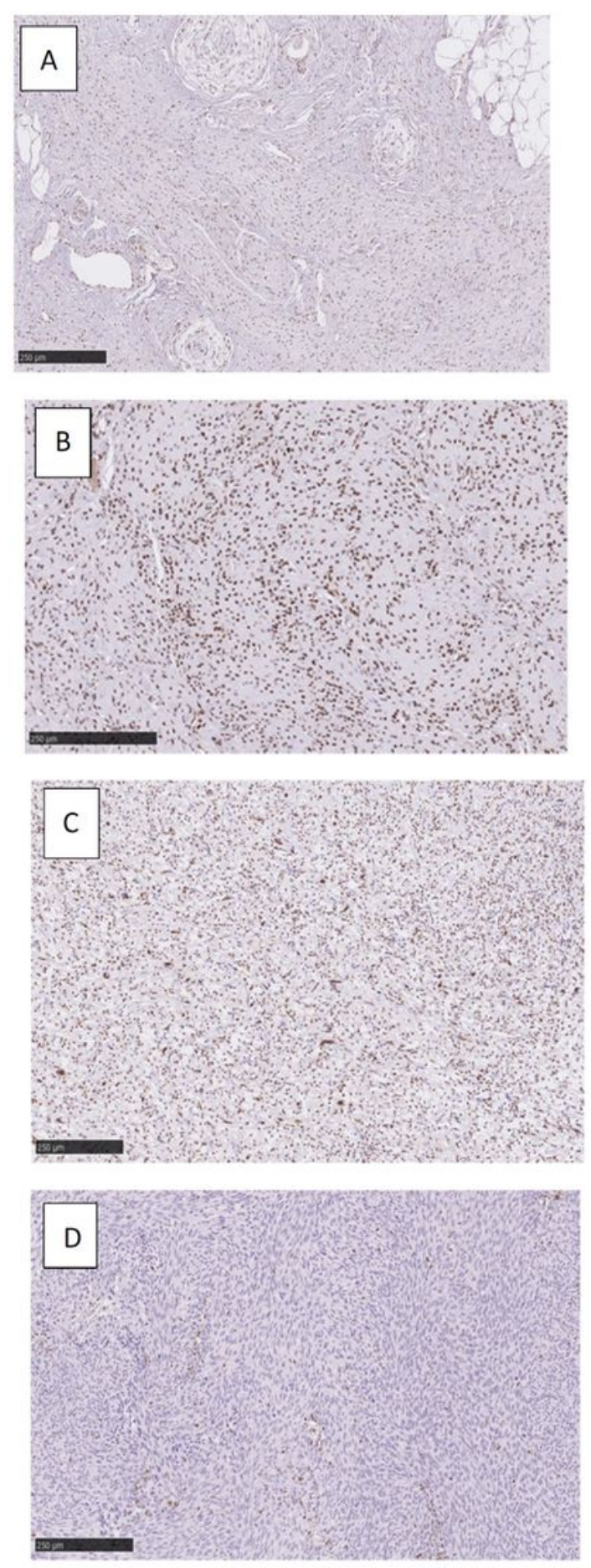

\section{Figure 3}

Immunohistochemical expression of H3K27me3 in NF1-associated tumors. A. neurofibroma, diffuse (+/-). B. neurofibroma, diffuse (fascicular pattern, +). C. Atypical neurofibroma (+). D. MPNST (-).

\section{Supplementary Files}


This is a list of supplementary files associated with this preprint. Click to download.

- Table3PRC2genealterationsandIHC.pdf

- Table2Detailsofhistopathology.pdf

- Table1NF1mutationandcopynumberalterations.pdf 